ESAIM: PROCEEDINGS, November 2002, Vol.12, 134-139

M.Thiriet, Editor

\title{
CHANGES IN FLUID MECHANICS AFTER ENDOVASCULAR STENTING : THE PRESSURE WAVES POINT OF VIEW
}

\author{
F. Nicoud, H. Vernhet \& M. Dauzat ${ }^{1}$
}

\begin{abstract}
A 1D model is presented which enables to assess the amount of wave reflexion due to the stenting of an elastic artery. It suggests that overdilated stents produce less reflexion, the optimal overdilation being proportional to the compliance ratio.
\end{abstract}

Résumé. Un modèle 1D permettant d'estimer le coefficient de reflexion induit par le placement d'un stent dans une artère élastique est présenté. Il suggère que les prothèses surdilatées produisent moins de reflexion, la surdilataion optimale étant proportionnelle au rapport des compliances.

\section{Motivation \& Objectives}

Angioplasty with or without endovascular stenting is a promising minimally invasive technique that can be used as treatment of occlusive disease in medium to large arteries. It has been applied extensively in the coronary, renal, and peripheral vascular systems with good results and low complication rates. The incidence of restenosis after angioplasty depends largely on the diameter of the vessel involved (its rate increases as the luminal diameter of the native vessel decreases). The use of intravascular stents tends to lower the complication rate although restenosis rates as high as 15-30 \% after 6 months for human coronary arteries have been observed, depending on the clinical series [1].

In vivo testings $[2-4]$ show that endovascular stenting induces a large modification of the arterial compliance and thus may modify drastically the propagation of arterial waves by introducing artificial reflexions. The first objective of this study is then to assess the amount of pressure wave reflexion related to the endovascular stenting of an elastic artery. For this purpose, the stented section of the vessel is modelled as an elastic duct whose compliance is less than the non-stented artery. We intent to clarify the extent of reflexion that can be expected depending on the characteristics of both the stent and the host vessel.

Typical differences in stent design concern the type and size of the wires, the shape of the structure, the length and surface of the stent, the radial strength and the longitudinal flexibility $[5,6]$. As far as pressure wave reflexion is concerned, the diameter of the stent and its effective compliance are key parameters. The second objective of the paper is to optimize the diameter of the prosthesis in such a way to minimize the amount of wave reflexion. For this purpose, we couple a model for the pressure wave evaluation to a steepest descent algorithm. The control parameter for this optimisation procedure is the global shape of the stent while its compliance remains fixed and homogeneous along its length.

The basic equations describing the pressure wave propagation are recalled in section 2 where a simple analytical expression for the reflexion coefficient is also provided. Results from the shape optimisation of the stent are then discussed in section 3.

\footnotetext{
1 University of Montpellier II - CC51 - ACSIOM Laboratory FRE 2311 and University of Montpellier I - Laboratory of Cardiovascular Physiology EA 2992
}

(c) EDP Sciences, SMAI 2003 


\section{FormALiSM}

\subsection{Basic equations}

The general one-dimensional equations describing the pulsatile blood flow (mass and momentum conservation) in compliant arteries are well known since the 70's [7]:

$$
\begin{aligned}
\frac{\partial A}{\partial t}+\frac{\partial A u}{\partial x} & =0 \\
\frac{\partial u}{\partial t}+u \frac{\partial u}{\partial x} & =-\frac{1}{\rho} \frac{\partial P}{\partial x}-\frac{f_{v} u}{\rho}
\end{aligned}
$$

where $u$ and $P$ are the averaged (over the cross section of the artery) velocity along the $x$-direction and relative $(P=0$ outside the duct) pressure respectively, $A$ is the area of the cross section, $\rho$ is the blood density and $f_{v} u / \rho$ stands for the viscous drag. Assuming that the velocity and pressure fluctuations are small enough to neglect non-linear terms and introducing the state equation of the artery $A=A(P)$, one is left with:

$$
\begin{array}{r}
\frac{\partial P}{\partial t}+\frac{A}{A^{\prime}} \frac{\partial u}{\partial x}=0 \\
\frac{\partial u}{\partial t}+\frac{1}{\rho} \frac{\partial P}{\partial x}+\frac{f_{v} u}{\rho}=0
\end{array}
$$

where $A^{\prime}$ stands for the pressure derivative of the cross section area, viz. the compliance. The non-linear formulation Eq. 1, although more general than Eq. 2, suffers from several drawbacks. Indeed, since it has to be solved in the time domain, the viscous drag term must be assessed by using the Poiseuille assumption for the velocity profile instead of the more accurate frequency dependant Womersley solution [8]. Moreover, any viscoelastic wall behavior is difficult to be accounted for since $A^{\prime}$ may depend on the frequency of the perturbation in this case. On the contrary, the linear formulation Eq. 2 can be solved in the Fourier space and the above mentioned physical behaviors (frequency dependant velocity profile and viscoelasticity) can be included without difficulty. Consistently, Reuderink et al. [9] have shown that a better overall accuracy is reached by using the linear formulation. In the course of the present study, $u$ and $P$ will be taken as solution of Eq. 2.

Considering a sector whose diameter and compliance do not depend on the space variable $x$ and letting $P=\hat{P} \exp (-j \omega t)$ and $u=\hat{u} \exp (-j \omega t)$, where $j^{2}=-1$ and $\omega$ is the wave pulsation, the classical wave equation $d^{2} \hat{P} / d x^{2}+k^{2} \hat{P}=0$ can be easily derived, the complex wave number being $k=\sqrt{\frac{A^{\prime}}{A} \omega\left(\rho \omega+j f_{v}\right)}$ and the wave speed being $c=\omega / k$. The general solution within a homogeneous segment is then :

$$
\begin{aligned}
\hat{P} & =P^{+} \exp \left(j k\left(x-x_{0}\right)\right)+P^{-} \exp \left(-j k\left(x-x_{0}\right)\right) \\
\hat{u} & =\frac{k}{\rho \omega+j f_{v}}\left(P^{+} \exp \left(j k\left(x-x_{0}\right)-P^{-} \exp \left(-j k\left(x-x_{0}\right)\right)\right)\right.
\end{aligned}
$$

where $x_{0}$ is the abscissa of the left boundary of the sector and $P^{+}$and $P^{-}$correspond to the amplitude of the forward and backward pressure waves. Their values are determined in order to satisfy the boundary conditions at $x=x_{0}$ and $x=x_{0}+L$ where $L$ is the length of the sector. 


\subsection{Modeling of the stenting}

In the purpose of modeling the wave reflexion induced by an endovascular stent placed in an elastic artery, three successive homogeneous segments are considered, each having its own set of constant area and compliance (see Fig. 1).

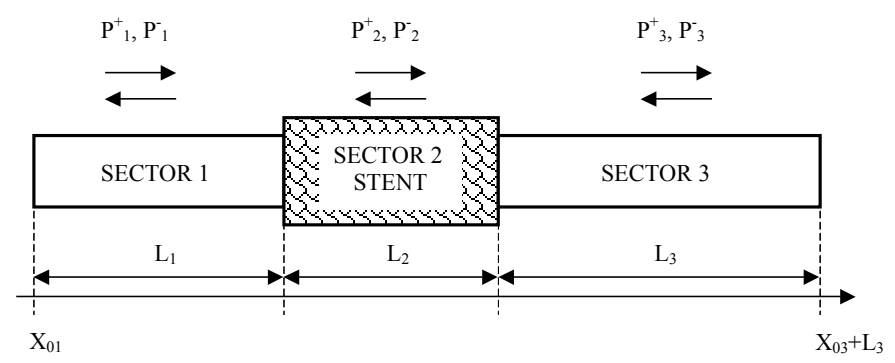

FIgURE 1. Schematic of the three homogeneous sectors used in order to model an artery stenting in terms of waves.

Each physical quantity in sector number $i(i=1,2,3)$ is denoted with indice $i$ (e.g. $\hat{u}_{1}$ and $L_{2}$ correspond to the velocity in sector 1 and the length of sector 2 respectively). Conservation of the total flow rate and energy at the interfaces $1-2$ and $2-3$ requires, for $j=1,2$ :

$$
A_{j} \hat{u}_{j}\left(x_{0_{j}}+L_{j}\right)=A_{j+1} \hat{u}_{j+1}\left(x_{0_{j+1}}\right) \quad, \quad \hat{P}_{j}\left(x_{0_{j}}+L_{j}\right)=\hat{P}_{j+1}\left(x_{0_{j+1}}\right)
$$

Two boundary conditions at $x=x_{0_{1}}=0$ and $x=x_{0_{3}}+L_{3}$ are needed in order to close the problem. In order to assess the stent response without spurious wave reflexion, non-reflecting boundary conditions are prescribed at both sides, leading to $P_{1}^{+}=1$ and $P_{3}^{-}=0$. The four remaining wave amplitudes, viz. $P_{2}^{+}, P_{3}^{+}, P_{1}^{-}, P_{2}^{-}$, are determined by solving Eqs. 4. The complex coefficient of wave reflexion due to the stent is then defined as $R_{\text {stent }}=\exp \left(-2 j k_{1} L_{1}\right) P_{1}^{-} / P_{1}^{+}$. After some algebra one finds out:

$$
R_{\text {stent }}=\frac{A_{2} K_{2}\left(A_{1} K_{1}-A_{3} K_{3}\right) \cos \left(k_{2} L_{2}\right)-j\left(A_{1} K_{1} A_{3} K_{3}-\left(A_{2} K_{2}\right)^{2}\right) \sin \left(k_{2} L_{2}\right)}{A_{2} K_{2}\left(A_{1} K_{1}+A_{3} K_{3}\right) \cos \left(k_{2} L_{2}\right)-j\left(A_{1} K_{1} A_{3} K_{3}+\left(A_{2} K_{2}\right)^{2}\right) \sin \left(k_{2} L_{2}\right)}
$$

where $K_{i}=k_{i} /\left(\rho \omega+j f_{v_{i}}\right)$. A typical value of the speed of propagation of waves in (human) arteries being a few meters per second, the wavelength is usually a few meters. On the other hand, the length scale of the stent is most likely equal to a few centimeters, meaning that the module of the dimensionless parameter $k_{2} L_{2}$ is small compared to unity. Moreover, since the goal of this study is to assess the wave reflexion related to the endovascular prosthesis, one can assume that zero reflexion occurs in absence of stent, viz. $A_{1} K_{1}=A_{3} K_{3}$ (in other words, we assume that host artery is perfectly homogeneous). Finally, one obtains the following first order expression for the reflexion coefficient:

$$
R_{\text {stent }} \simeq \frac{j\left(1-\Lambda^{2}\right)}{2 \Lambda} k_{2} L_{2}, \quad \Lambda=\frac{A_{1} K_{1}}{A_{2} K_{2}}
$$

This relation shows that the theoretical reflexion induced by an endovascular prosthesis decreases with the length of the stent and increases with the pulsation of the wave. Moreover the reflexion coefficient is zero as soon as $\Lambda=1$, viz. $A_{1} K_{1}=A_{2} K_{2}$. Assuming that viscous effects can be neglected in the reflexion process makes $\Lambda$ and $k_{2}$ real numbers with $\Lambda=\sqrt{A_{1} A_{1}^{\prime} / A_{2} A_{2}^{\prime}}$ and $k_{2} L_{2}=\omega \sqrt{\rho A_{2}^{\prime} / A_{2}} L_{2}$. Eq. 6 leads then to a convenient formula to assess the amount of wave reflexion :

$$
R_{\text {stent }} \simeq j \omega \sqrt{\rho} \frac{A_{2} A_{2}^{\prime}-A_{1} A_{1}^{\prime}}{2 \sqrt{A_{1} A_{1}^{\prime}}} \frac{L_{2}}{A_{2}}
$$



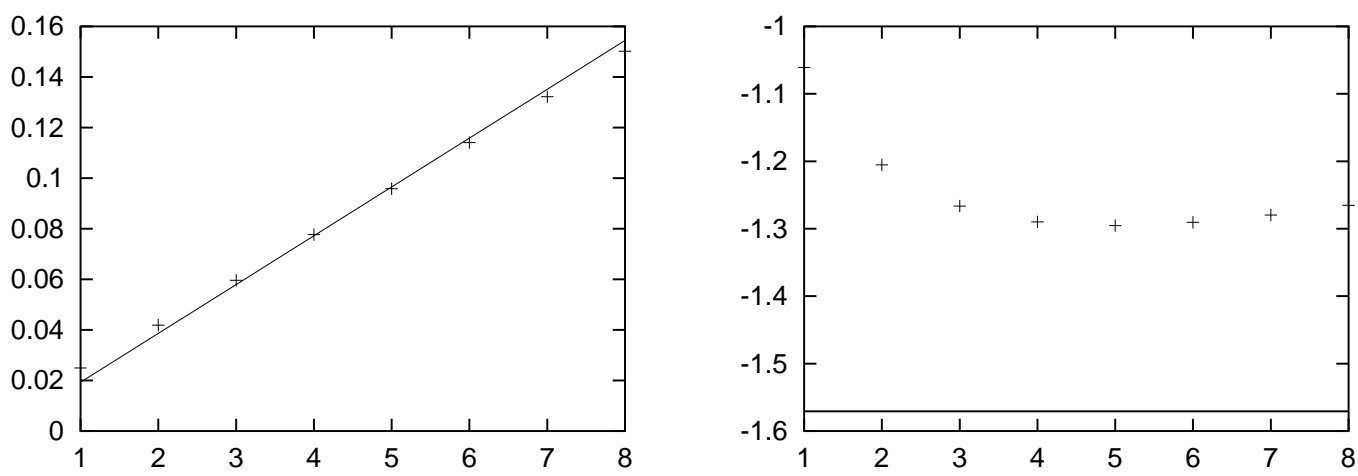

FiguRE 1. Reflexion coefficient for the first eigth modes (fundamental is $\omega=8 \pi$ ) obtained by the full (solid line, Eq. 5) and approximated (symbols, Eq. 7) 1D models. The physiological data are from Vernhet et al. [2] (see text). Left side: modulus, Right side: phase (rad)

From this relation, the amount of wave reflexion is related to the geometrical/mechanical mismatch induced by the stenting, a stent shape factor $L_{2} / A_{2}$ as well as the flow conditions. Moreover, it shows that a stent satisfying the relation $A_{2}=A_{1} A_{1}^{\prime} / A_{2}^{\prime}$ produces no wave reflexion. The compliance $A_{2}^{\prime}$ of the stent being always smaller than the compliance $A_{1}^{\prime}$ of the host artery, it follows that overdilation $\left(A_{2}>A_{1}\right)$ tends to reduce the amount of reflected waves. Finally, in absence of viscous effects, $\Lambda$ and $k_{2}$ are real numbers and it follows from Eq. 6 that the phase difference between the reflected wave and the incident one is $-\pi / 2$.

\subsection{Results - Discussion}

In order to illustrate the previous theoretical formulae, we present some results obtained for a realistic set of conditions. The mechanical and geometrical data were obtained from animal experimentations (rabbits sub-renal aorta) performed by Vernhet et al. [2]: $r_{1}=r_{2}=1.5 \mathrm{~mm}, A_{1}^{\prime} / A_{1}=A_{3}^{\prime} / A_{3}=20,7.10^{-6} \mathrm{~Pa}^{-1}$, $A_{2}^{\prime} / A_{2}=3,7.10^{-6} \mathrm{~Pa}^{-1}$. The results presented are for $f_{v}=8 \pi \mu / A_{i}$ where $i=1,2,3$ and $\mu$ is the dynamic viscosity of blood, $\mu=0.01 \mathrm{Pa.s}^{-1}$. This expression for $f_{v}$ is derived by assuming that the velocity profile is parabolic. The stent is $13 \mathrm{~mm}$ long. The reflection coefficient for the first eight modes is shown in figure 1. Note that the fundamental frequency is $4 \mathrm{~Hz}$, in agreement with the data. Both the modulus and the phase of $R_{\text {stent }}$ as estimated from Eq. 7 are in good agreement with the exact form Eq. 5. For the present data, the modulus of the reflexion coefficient is a few percent but larger values would be reached for larger and more compliant vessels, especially for longer stents. Wave reflexions affect the blood flow through a vessel by modifying the pressure distribution within the artery. For example, in the case of a forward pressure wave of amplitude $P^{+}$ and wave number $k$, the pressure distribution the pressure distribution is $\hat{P}=P^{+} \exp (j k x)$ (assuming that the abscissa of the most proximal section is 0 ). If a stent is placed at abscissa $L$, the pressure distribution upstream the prosthesis becomes $\hat{P}=P^{+}\left(\exp (j k x)+R_{\text {stent }} \exp (-j k(x-2 L))\right)$. Thus, the modification in the pressure field is proportional to the reflexion coefficient and virtually extends throughout the whole vascular system. Note that under in vivo conditions, wave reflexions naturally occur at each branching/bifurcation of the vascular tree. The effect of these reflexions accumulate in a hierarchic way from one level of the tree to the next. The pressure field in the vascular system results from the pressure waves generated at the heart level as well as the way these waves propagate and are reflected within the vessels. The stenting introduces an artificial site of reflexion whose effect on the global pressure field is both unclear and beyond the scope of this paper. 


\section{Optimisation of the Stent Shape}

In the previous section, the stent was modeled as a single homogeneous sector with constant area and compliance. Consequently, minimizing the amount of wave reflexion means introducing a geometry discontinuity to balance the compliance mismatch induced by the stenting. We now investigate the possibility to reduce the wave reflexion without introducing geometrical discontinuities. For this purpose, the stent is made inhomogeneous in space and is modeled thanks to $N$ successive homogeneous sectors. Each of these sectors has its own area and compliance. The whole stented artery is now represented by $N+2$ sectors and $2(N+2)$ unknowns while $2(N+1)$ jump equations (see Eq. 4) as well as two non-reflecting boundary conditions at $x=0$ and $x=x_{0_{N+2}}+L_{N+2}$ must be satisfied. The corresponding linear system is now solved numerically.

The length of the $N$ sectors modeling the prosthesis is $L_{\text {stent }} / N$ and we suppose that they all share the same compliance value. Their cross section areas are not fixed a priori but optimized thanks to a steepest descent algorithm, the cost function being simply the modulus of the reflexion coefficient $R_{\text {stent }}$. In order to manage the regularity of the solution, the shape of the stent is parameterized thanks to a polynomial function of order $d$. Namely, the radius $r_{j}$ of sector number $j, 1<j<N+2$, is written as $r_{j}=r_{1}+\sum_{p=0}^{d} \alpha_{p} x_{j}^{p}$, where $x_{j}=x_{0_{j}}+L_{j} / 2$ is the abscissa of the middle of the $j^{\text {th }}$ sector and $r_{1}$ is the fixed radius of the host artery before stenting $\left(r_{1}=r_{N+2}=\sqrt{A_{1} / \pi}\right)$. Besides enforcing the regularity of the solution, this parameterization reduces the length of the control vector from $N$ to $d+1$ (typical values were $d=4$ and $N=20$ ). The regularity of the transition between the host artery and the stented sector is imposed by stating that the polynomial function and its first derivative are zero at the edges of the stent.

Fig. 2 shows the evolution of the cost function during a typical optimization run. As in section 2.3, the mechanical and geometrical data were obtained from animal experimentations performed by Vernhet et al. [2]: $\omega=8 \pi$ (only the fundamental frequency is considered here), $r_{1}=r_{N+2}=1.5 \mathrm{~mm}, A_{1}^{\prime} / A_{1}=A_{N+2}^{\prime} / A_{N+2}=$ 20,7.10 ${ }^{-6} \mathrm{~Pa}^{-1}, A_{j}^{\prime} / A_{j}=3,7.10^{-6} \mathrm{~Pa}^{-1}, 1<j<N+2$. The results presented are for $f_{v}=0$. The stent is $13 \mathrm{~mm}$ long and is modeled with $N=26$ sectors of length $0.5 \mathrm{~mm}$. The shape is parameterized with a fourth order polynomial function $(d=4)$. After 600 iterations the normalized cost function is close to zero, meaning that the new shape of the stent is much less reflecting than the initial one (which consisted in a straight duct of radius $r_{1}$ ). The optimized shape of the prosthesis is shown in Fig. 3. The solution meets the regularity constraints imposed at both edges of the stent. It recovers the trend observed in section 2 since the diameter in the middle part of the prosthesis is larger than that of the host artery.

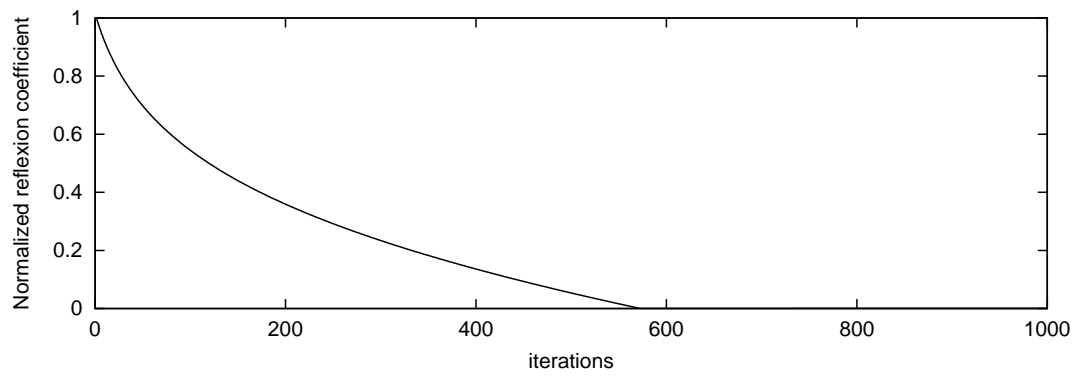

Figure 2. Evolution of the normalized coefficient of wave reflexion due to stenting (in module).

\section{Conclusion}

The theoretical results discussed in this paper show that the reflexion coefficient is proportional to the stent-to-wavelength ratio. Thus, it is most likely that the amount of wave reflexion remains rather small. An easy-to-use formula is provided in order to assess the reflexion coefficient from the knowledge of the compliance 


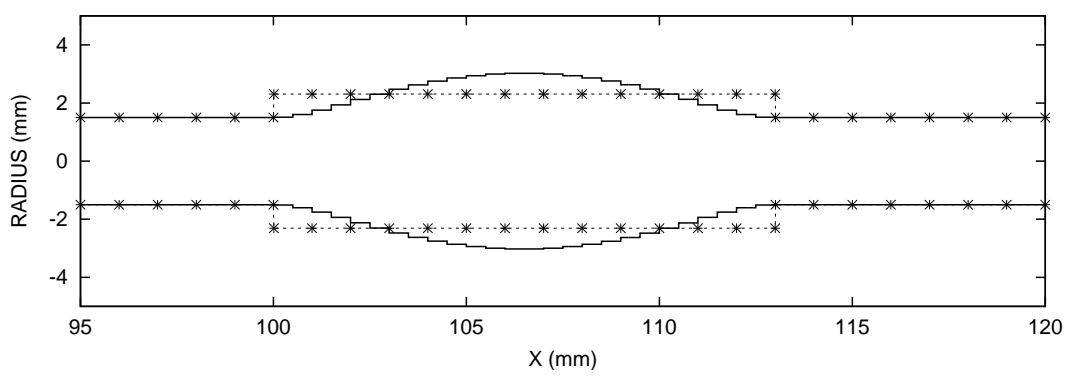

Figure 3. Typical optimized shape design for the reduction of the pressure waves reflexion. Symbols correspond to the analytical solution for an homogeneous stent (see section 2).

before and after stenting. The global shape optimization of the endovascular prosthesis performed from the 1D model allows to eliminate the major part of the pressure wave reflexion related to the compliance mismatch. This theoretical study suggests that overdilated stents produce less reflexion, the optimal overdilation being proportional to the compliance ratio. Under in vivo conditions, however, the efficiency of stenting does not depend only on the wave reflexion and other effects must be accounted for. Regarding stent overdilation, contradictory findings are reported in the literature. From their angiographic results, Caixeta et al. [10] deduce that overdilation is not responsible for higher restenosis rate while inducing a larger patent arterial lumen. On the other hand, Koyama et al. [11] argue that the vascular overstretch caused by stent overdilation promotes intimal hyperplasia. Recent in vivo testing on 18 rabbits sub renal aorta (Vernhet et al., under consideration for publication) show that a $130 \%$ overdilation increases the intimal thickening phenomena by $20-30 \%$ and multiplies an injury score [12] by more than three, from 0.4 to 1.4. Consequently, the optimal (as far as wave reflexion is concerned) stent diameter suggested by Eq. 7, viz. $A_{2}=A_{1} A_{1}^{\prime} / A_{2}^{\prime}$, would probably lead to strong wall mechanics injuries. However, it is likely that an optimum overdilation rate exists, which limits the amount of wave reflexion (or more generally hemodynamic perturbations) without inducing unacceptable vascular stretching. Further studies are necessary to clarify this issue.

\section{REFERENCES}

[1] T. Rau, J. Schofer, M. Schluter, A. Seidensticker, J. Berger, and D.G. Mathey. Stenting of non acute total coronary occlusions: predictors of late angiographic outcome. J. Am. Coll. Card., 31:275-280, 1998.

[2] H. Vernhet, R. Demaria, M.C. Oliva-Lauraire, J.M. Juan, J.P. Sénac, and M. Dauzat. Changes in wall mechanics after endovascular stenting in rabbit aorta: comparison of three different stent designs. Am. J. Roent., 176(3):803-807, 2001.

[3] H. Vernhet, J.M. Juan, R. Demaria, M.C. Oliva-Lauraire, J.P. Sénac, and M. Dauzat. Acute changes in aortic wall mechanical properties after stent placement in rabbits. J. Vasc. Inter. Rad., 11(5):634-638, 2000.

[4] P.H. Rolland, A.B. Charifi, and C. Verrier. Hemodynamics and wall echanics after stent placement in swine illiac arteries: comparative results from six stent designs. Radiology, 213:229-246, 1999.

[5] G. Tepe, SH. Duda, and H. Hanke. Covered stents for prevention of restenosis. Invest. Radiol, 4:223-229, 1996.

[6] C. Rogers, DY. Tseng, JC. Squire, and ER. Edelman. Balloon-artery interactions during stent placement. a finite element analysis approach to pressure, compliance and stent design as contributors to vascular injury. Circ. Res., 84:378-383, 1999.

[7] T. Hughes and J. Lubliner. On the 1D theory of blood flow in the larger vessels. Math. Biosciences, 18:161-170, 1973.

[8] J.R. Womersley. Oscillatory motion of a viscous liquid in a thin-walled elastic tube- i: The linear approximation for long waves. Philosophical Magazine (series 7), 46:199-221, 1955.

[9] PJ. Reuderink, HW. Hoogstraten, P. Sipkema, B. Hillen, and N. Westerhof. Linear and nonlinear one-dimensional models of pulse wave transmission at high womersley numbers. J. Biomech., 22:819-827, 1989.

[10] A.M. Caixeta, F.S. Jr. Brito, and M. Rati. High versus low-pressure balloon inflation during multilinktrade mark stent implantation: acute and long-term angiographic results. Catheter Cardiovasc. Interv., 50:398-401, 2000.

[11] J. Koyama, M. Owa, and S. Sakurai. Relation between vascular morphologic changes during stent implantation and the magnitude of in-stent neointimal hyperplasia. Am. J. Cardiol., 86:753-758, 2000.

[12] R.S. Schwartz, K.C. Huber, and J.G. Murphy. Restenosis and the proportional neointimal response to coronary artery injury: results in a porcine model. J. Am. Coll. Cardiol., 19:267-274, 1992. 